\title{
Article \\ Co-Infections in Critically Ill Patients with or without COVID-19: A Comparison of Clinical Microbial Culture Findings
}

\author{
Rosario Cultrera 1,*(D), Agostino Barozzi ${ }^{2}$, Marco Libanore ${ }^{3}$, Elisabetta Marangoni ${ }^{4}$, Roberto Pora ${ }^{2}$, \\ Brunella Quarta ${ }^{5}$, Savino Spadaro ${ }^{4}$, , Riccardo Ragazzi ${ }^{4}$, Anna Marra ${ }^{5}$, Daniela Segala ${ }^{1}$ \\ and Carlo Alberto Volta ${ }^{4}$ (D)
}

\section{check for} updates

Citation: Cultrera, R.; Barozzi, A.; Libanore, M.; Marangoni, E.; Pora, R.; Quarta, B.; Spadaro, S.; Ragazzi, R.; Marra, A.; Segala, D.; et al.

Co-Infections in Critically Ill Patients with or without COVID-19: A Comparison of Clinical Microbial Culture Findings. Int. J. Environ. Res. Public Health 2021, 18, 4358. https:// doi.org/10.3390/ijerph18084358

Academic Editor: Paul B. Tchounwou

Received: 1 March 2021

Accepted: 15 April 2021

Published: 20 April 2021

Publisher's Note: MDPI stays neutral with regard to jurisdictional claims in published maps and institutional affiliations.

Copyright: (c) 2021 by the authors Licensee MDPI, Basel, Switzerland. This article is an open access article distributed under the terms and conditions of the Creative Commons Attribution (CC BY) license (https:// creativecommons.org/licenses/by/ $4.0 /)$.
1 Infectious Diseases, Department of Traslational Medicine, University of Ferrara, 44121 Ferrara, Italy; daniela.segala@unife.it

2 Clinical Microbiology, Department of Biotechnology, Transfusional and Laboratory, University Hospital of Ferrara, 44124 Ferrara, Italy; a.barozzi@ospfe.it (A.B.); roberto.pora@ospfe.it (R.P.)

3 Infectious Diseases Unit, Department of Medicine, University Hospital of Ferrara, 44124 Ferrara, Italy; m.libanore@ospfe.it

4 Intensive Care Unit, Department of Traslational Medicine, University of Ferrara, 44121 Ferrara, Italy; e.marangoni@ospfe.it (E.M.); spdsvn@unife.it (S.S.); rgc@unife.it (R.R.); vlc@unife.it (C.A.V.)

5 Pharmacy Service, University Hospital of Ferrara, 44124 Ferrara, Italy; b.quarta@ospfe.it (B.Q.); a.marra@ospfe.it (A.M.)

* Correspondence: ctr@unife.it; Tel.: +39-0532239145 or +39-3473628614

\begin{abstract}
Co-infections in critically ill patients hospitalized for severe acute respiratory syndrome coronavirus 2 (SARS-CoV-2) infection have an important impact on the outcome of coronavirus disease 2019 (COVID-19). We compared the microbial isolations found in COVID-19 patients hospitalized in an intensive care unit (ICU) with those in a non-COVID-19 ICU from 22 February to 30 April 2020 and in the same period of 2019. We considered blood, urine or respiratory specimens obtained with bronchoalveolar lavage (BAL) or bronchial aspirate (BASP), collected from all patients admitted in ICUs with or without COVID-19 infection. We found a higher frequency of infections due to methicillin-resistant (MR) staphylococci, vancomycin-resistant Enterococcus faecium, carbapenemresistant Acinetobacter baumannii and Candida parapsilosis in COVID-19-positive patients admitted in ICUs compared to those who were COVID-19 negative. Carbapenem-resistant Pseudomonas aeruginosa was more frequently isolated from patients admitted in non-COVID-19 ICUs. Several conditions favor the increased frequency of these infections by antibiotic-resistant microorganisms. Among all, the severity of the respiratory tracts was definitely decisive, which required assisted ventilation with invasive procedures. The turnover in the ICU of a large number of patients in a very short time requiring urgent invasive interventions has favored the not always suitable execution of assistance procedures. No less important is the increased exposure to infectious risk from bacteria and fungi in patients with severe impairment due to ventilation. The highest costs for antifungal drugs were shown in the ICU-COVID group.
\end{abstract}

Keywords: SARS-CoV-2; COVID-19; co-infection; microbial culture; antimicrobial consumption; antimicrobial expenditure; intensive care unit

\section{Introduction}

Patients admitted to intensive care units (ICUs) are exposed to a greater risk of secondary infectious complications that more frequently affect the respiratory, urinary and circulatory systems, constituting one of the main causes of healthcare-associated infections (HAIs). These infections are often due to multi-drug-resistant microorganisms that require the use of next-generation antimicrobials with a serious impact on antimicrobial 
stewardship programs, on the appropriateness of the use of antibiotics and on a pharmacoeconomic level. The microbial analyses performed on these patients also contribute to the increase in healthcare costs, and are often performed in large quantities and not always necessary, resulting in their misinterpretation. In fact, the complicated distinction between colonization, contamination of the biological sample taken and infection often causes an overestimation of infections with consequent inappropriate antibiotic therapy. Bacterial and fungal co-infections can be complications of viral respiratory diseases such as seasonal/pandemic influenza [1], Middle East respiratory syndrome coronavirus (MERS$\mathrm{CoV}$ ) [2] and SARS-CoV-1 [3,4], and lead to an increase in outcome severity and to the death of individuals with or without pre-existing respiratory diseases.

The clinical manifestations of coronavirus disease 2019 (COVID-19) range from asymptomatic infection to severe viral pneumonia requiring treatment in an intensive care unit (ICU) [5-7]. The most common symptoms of COVID-19 are fever and cough and, due to serious respiratory disease, some patients need to be hospitalized and mechanical ventilation [6]; more severe outcomes [8] are associated with older age, a higher percentage of comorbidities and higher mortality $[9,10]$.

SARS-CoV-2 can directly damage the lung epithelium and indirectly ignite an aberrant cytokine storm, eventually leading to multi-organ failure [11,12]. To reverse this dysregulated activation of the immune system, immunosuppressive drugs are widely used [12,13]. A combination of virus- and drug-induced immunosuppression likely increases the susceptibility to secondary infections.

Some patients with COVID-19 need to be hospitalized due to severe respiratory complications and, in severe cases, undergo intensive care with supportive mechanical ventilation. Bacterial and fungal co-infections in critically ill patients hospitalized for SARS-CoV-2 infection have an important impact on the outcome of COVID-19 disease, with increased morbidity and mortality [14-16].

There are numerous studies on the empirical use of antibiotics in hospitalized patients with COVID-19 [17,18], often induced by the evidence of inflammatory markers such as procalcitonin and C-reactive protein, which are normally associated with bacterial infections, even when they are not proven [19,20].

Our study examined microbial cultures performed in COVID-19-positive patients admitted to an ICU from 22 February 2020 to 30 April 2020. The results were compared with microbial cultures performed in hospitalized non-COVID-19 patients in another ICU of the same hospital from 22 February 2020 to 30 April 2020 and in 2019, prior to the pandemic spread of COVID-19. We also analyzed the consumption of antibiotics in these ICUs in the same periods.

The primary objectives were to define (i) the number of microbial cultures of blood, urine and bronchoaspirate/bronchoalveolar lavage samples, (ii) the frequency of isolated bacterial and fungal species, (iii) the consumption of antimicrobials in ICUs in the two periods examined and in patients with or without COVID-19.

The secondary objective was to evaluate the expenditure on antimicrobials used by each ICU in relation to the infections identified by culture tests.

\section{Materials and Methods}

\subsection{Study Population}

We considered blood, urine or respiratory specimens obtained with bronchoalveolar lavage (BAL) or, when it was not available, bronchial aspirate (BASP), collected from all patients admitted in intensive care units (ICUs) with (ICU-COVID) or without COVID-19 infection (ICU-noCOVID) from 22 February to 30 April 2020 to study the microbiological findings. Moreover, we considered the same specimens collected in the ICU (ICU-2019) from 22 February to 30 April 2019, before the pandemic.

The ICUs were mixed medical-surgical ICUs (ICU-noCOVID and ICU-2019), and a dedicated ICU for COVID-19-positive patients (ICU-COVID) at University Hospital of 
Ferrara, Italy. All patients admitted in these ICU wards needed mechanical ventilation settings, including constant-flow controlled ventilation.

COVID-19 was defined as the presence of a positive real-time polymerase chain reaction (RT-PCR) for SARS-CoV-2 in at least one respiratory specimen (nasopharyngeal swab, sputum, and/or lower respiratory tract specimens), associated with suggestive signs, symptoms and/or radiological findings.

Bloodstream infection (BSI) was defined as the presence of at least one positive blood culture for bacteria or fungi, drawn $>48 \mathrm{~h}$ after ICU admission. At least two consecutive positive blood cultures were needed to define BSI due to coagulase-negative staphylococci or other common skin colonizers (e.g., coagulase-negative staphylococci, non-diphtheritic Corynebacteria, Bacillus spp., Propionibacterium spp., etc.) [21]. Patients who had more than one positive blood culture within 7 days from the first positive blood culture were considered to have a single episode of BSI with multiple isolates. Polymicrobial infections were considered as separate BSI events, one for each causative organism isolated from blood culture.

Positive cultures of potentially pathogenic organisms from the lower respiratory tract were defined as positive cultures of a respiratory specimen obtained with BAL or, when not available, BASP, excluding Candida spp.

Microbiologically, catheter-associated urinary tract infection (CA-UTI) is defined by microbial growth of $\geq 10^{3}$ colony-forming units (CFU)/mL of one or more bacterial species in a single catheter urine specimen or in a midstream voided urine specimen from a patient whose urethral, suprapubic or condom catheter has been removed within the previous $48 \mathrm{~h}$. All criteria to confirm CA-UTI in several clinical conditions and with particular microbiological findings were considered according to international classifications and reports [22-24].

Infection versus colonization was distinguished according to definitions reported by the European Centre for Disease Prevention and Control [25,26].

Each isolate was identified by matrix-assisted laser desorption ionization time-offlight (MALDI-TOF) by a VITEK ${ }^{\circledR}$ MS (bioMerieux). Antibiotic susceptibility testing was performed by a VITEK ${ }^{\circledR} 2$ instrument (bioMerieux), with Card AST-N376 for Gramnegative bacteria screening and N397 to test the susceptibility of Enterobacteriales to cephalosporins / $\beta$-lactamase inhibitor combinations, Card AST-P659 for staphylococci, Card AST-P658 for enterococci, Card AST-ST03 for streptococci (S. pneumoniae, betahemolytic Streptococcus and viridans Streptococcus) and Card AST-YS08 for clinically significant yeasts.

Methicillin-resistant Staphylococcus aureus (MRSA) and vancomycin-resistant Enterococci (VRE) strains were detected by cefoxitin screening and an oxacillin MIC test with Card AST-P659 and a teicoplanin/vancomicin MIC test with Card AST-P658, respectively.

MIC values were interpreted according to current European Committee on Antimicrobial Susceptibility Testing (EUCAST) clinical breakpoints.

Carbapenemase-producing Enterobacteriales (CPE) strains were confirmed by microdilution (Sensititre ${ }^{\mathrm{TM}}$, Thermo Fisher Scientific) EURGNCOL and DKMGN plates. Phenotypical CPE resistance was confirmed by synergic test diffusion Diatabs ${ }^{\mathrm{TM}}$ (Rosco Diagnostica) on Mueller-Hinton agar (Vacutest-Kima). A genotyping test for CPE resistance was performed by RT-PCR (GeneXpert ${ }^{\circledR}$ ) with an Xpert ${ }^{\circledR}$ Carba-R test (Cepheid Inc., Sunnyvale, CA, USA). MIC values were interpreted according to current European Committee on Antimicrobial Susceptibility Testing (EUCAST) clinical breakpoints.

Blood, respiratory and urine cultures were requested by the attending physicians for patients with suspected secondary infections because of clinical and/or respiratory deterioration associated with suggestive laboratory or radiological findings.

\subsection{Antimicrobial Consumption and Costs}

The data of the antibiotic consumption were normalized to DDD/100 bed days for inpatients. This information was calculated independently by the pharmacy service, based 
on dispensing rather than administration data, using our own computer system that estimated the DDD [27].

Crude costs of antimicrobial drugs were collected from the administrative pharmacy data.

\subsection{Statistical Analyses}

We described the microbial culture carried out in ICU wards with or without COVID19-positive patients between 6 March and 30 April 2020 compared to the microbial tests done in same ward during the same period in 2019. Descriptive analyses were performed for all variables. Count and proportion were presented for all categorical variables.

The primary outcomes were the number of microbial cultures and the frequency of bacterial and fungal species isolated in ICUs with or without COVID-19 positive patients, and to define the consumption of antimicrobials in these wards.

The secondary aim was to evaluate a possible higher expenditure on antimicrobials in ICUs during the pandemic.

Bivariate comparisons using chi-square (or Fisher's exact) tests were conducted for nominal data and two-sample $t$-tests or Mann-Whitney $U$ tests for continuous data (depending on normality distribution) were used to compare characteristics and outcomes between the samples of patients. A $p$ value $<0.05$ was accepted as statistically significant.

\section{Results}

The analyses performed show an increase in the number of microbial cultures per patient done in the ICU-COVID (20.5/patient) group compared to the number of analyses made in the same period in the ICU-noCOVID group (7/patient) and in ICU-2019 group (8.3/patient), before the SARS-CoV-2 pandemic (Table 1$)$. The clear difference remains even after subtracting from the count the double negative samples on the same day (ICUCOVID: 14.5/patient; ICU-noCOVID: 4.4/patient; ICU-2019: 5.5/patient) (Table 2). There was an increase in positive blood cultures compared to the other two cohorts, while positive culture differences were not found for bronchoaspirate, broncholavage and urine cultures (Table 1).

Table 1. Microbial cultures in patients.

\begin{tabular}{|c|c|c|c|c|c|c|c|}
\hline & \multirow{2}{*}{\multicolumn{2}{|c|}{$\begin{array}{c}\text { ICU-2019 } \\
\text { Patients }(n=58)\end{array}$}} & \multirow{2}{*}{\multicolumn{2}{|c|}{$\begin{array}{l}\text { ICU-noCOVID } \\
\text { Patients }(n=47)\end{array}$}} & \multirow{2}{*}{\multicolumn{2}{|c|}{$\begin{array}{c}\text { ICU-COVID } \\
\text { Patients }(n=28)\end{array}$}} & \multirow{3}{*}{$p$} \\
\hline & & & & & & & \\
\hline & TOT & POS (\%) & TOT & POS (\%) & TOT & POS (\%) & \\
\hline Central venous blood culture & 178 & $26(14.6)$ & 119 & $18(15.1)$ & 206 & $71(34.5)$ & $<0.05$ \\
\hline Periferal venous blood culture & 160 & $21(13.1)$ & 122 & $12(9.8)$ & 198 & $48(24.3)$ & $<0.05$ \\
\hline Bronchoaspirate culture & 103 & $52(50.5)$ & 72 & $40(55.6)$ & 134 & $82(61.2)$ & N.S. \\
\hline Bronchoalveolar lavage culture & 15 & $7(46.7)$ & 8 & $4(50)$ & 9 & $6(66.7)$ & N.S. \\
\hline Urinary catheter & 25 & $10(40)$ & 7 & $4(57.1)$ & 27 & $13(48.1)$ & N.S. \\
\hline Tot. & 481 & $116(24.1)$ & 328 & $78(23.8)$ & 574 & $220(38.3)$ & $<0.05$ \\
\hline
\end{tabular}

N.S.: not significant, $p>0.05$.

Table 2. Gram-positive and Gram-negative bacteria and fungal isolates.

\begin{tabular}{|c|c|c|c|c|c|c|c|c|c|}
\hline & \multicolumn{3}{|c|}{ ICU-2019 } & \multicolumn{3}{|c|}{ ICU-noCOVID } & \multicolumn{3}{|c|}{ ICU-COVID } \\
\hline & \multicolumn{3}{|c|}{ Patients $(n=58)$} & \multicolumn{3}{|c|}{ Patients $(n=47)$} & \multicolumn{3}{|c|}{ Patients $(n=28)$} \\
\hline & Gram+ & Gram- & Fungi & Gram+ & Gram- & Fungi & Gram+ & Gram- & Fungi \\
\hline Central venous blood culture & 21 & 4 & 1 & 13 & 5 & 0 & 43 & 16 & 12 \\
\hline Periferal venous blood culture & 16 & 5 & 0 & 10 & 2 & 0 & 32 & 10 & 6 \\
\hline Bronchoaspirate culture & 3 & 46 & 3 & 3 & 17 & 20 & 9 & 50 & 23 \\
\hline Bronchoalveolar lavage culture & 2 & 5 & 0 & 1 & 1 & 2 & 3 & 3 & 0 \\
\hline Urinary catheter & 2 & 7 & 1 & 0 & 1 & 3 & 2 & 2 & 9 \\
\hline Tot. & 44 & 67 & 5 & 27 & 26 & 25 & 89 & 81 & 50 \\
\hline
\end{tabular}


Taking into consideration the different classes of isolated microorganisms, an increase in fungal infections in ICU-COVID emerges compared to the other two cohorts: Candida albicans $(n=29)$, C. parapsilosis $(n=13)$. The most frequently isolated bacteria were Acinetobacter baumannii, Enterococcus faecalis and E. faecium, Staphylococcus epidermidis and Stenotrophomonas maltophilia (Table 3). No relevant data emerged regarding the number of positive cultures for MRSA, extended spectrum $\beta$-lactamase (ESBL)-positive and carbapenem-resistant and carbapenemase-producing Enterobacteriaceae in the three cohorts (Table 4).

Table 3. Bacterial and fungal isolates in patients admitted in three ICUs.

\begin{tabular}{|c|c|c|c|c|}
\hline & ICU-2019 & ICU-noCOVID & ICU-COVID & \multirow{2}{*}{$p$} \\
\hline & Patients $(n=58)$ & Patients $(n=47)$ & Patients $(n=28)$ & \\
\hline Microbial Isolates & $n$ & $n$ & $n$ & \\
\hline Acinetobacter baumannii & 9 & - & 17 & $<0.05$ \\
\hline Candida albicans & - & 11 & 29 & $<0.05$ \\
\hline Candida glabrata & 2 & 5 & 4 & N.S. \\
\hline Candida krusei & - & 1 & - & N.S. \\
\hline Candida lusitaniae/Ciavispora I & 2 & - & 2 & N.S. \\
\hline Candida parapsilosis & - & - & 13 & N.S. \\
\hline Candida tropicalis & 1 & 8 & - & N.S. \\
\hline Enterococcus faecalis & 2 & 1 & 14 & $<0.05$ \\
\hline Enterococcus faecium & 1 & 1 & 10 & $<0.05$ \\
\hline Pseudomonas aeruginosa & 15 & 9 & 3 & N.S. \\
\hline Staphylococcus epidermidis & 21 & 10 & 42 & $<0.05$ \\
\hline Stenotrophomonas maltophilia & 3 & 5 & 13 & $<0.05$ \\
\hline Total Samples Pos. & 116 & 78 & 120 & $<0.05$ \\
\hline
\end{tabular}

N.S.: not significant, $p>0.05$.

Table 4. Resistant bacteria isolated in three cohorts.

\begin{tabular}{|c|c|c|c|c|c|c|c|c|c|}
\hline & \multicolumn{3}{|c|}{ ICU-2019 } & \multicolumn{3}{|c|}{ ICU-noCOVID } & \multicolumn{3}{|c|}{ ICU-COVID } \\
\hline & \multicolumn{3}{|c|}{ Patients $(n=58)$} & \multicolumn{3}{|c|}{ Patients $(n=47)$} & \multicolumn{3}{|c|}{ Patients $(n=28)$} \\
\hline & MRSA & CPE/CRE & ESBL+ & MRSA & CPE/CRE & ESBL+ & MRSA & CPE/CRE & ESBL+ \\
\hline Central venous blood culture & 1 & 0 & 1 & 0 & 0 & 0 & 0 & $1 / 1$ & 0 \\
\hline Periferal venous blood culture & 0 & 0 & 1 & 0 & 0 & 0 & 0 & 0 & 0 \\
\hline Bronchoaspirate culture & 2 & 0 & 0 & 2 & $1 / 1$ & 0 & 2 & $2 / 2$ & 0 \\
\hline Bronchoalveolar lavage culture & 1 & 0 & 0 & 0 & 0 & 0 & 3 & 0 & 0 \\
\hline Urinary catheter & 0 & 0 & 0 & 0 & 0 & 0 & 0 & $1 / 1$ & 0 \\
\hline Tot. & 4 & 0 & 2 & 2 & $1 / 1$ & 0 & 5 & $4 / 4$ & 0 \\
\hline
\end{tabular}

MRSA, methicillin-resistant Staphylococcus aureus; CPE, carbapenemase-producing Enterobacteriales; CRE, carbapenem-resistant Enterobacteriales; ESBL, extended spectrum $\beta$-lactamase.

Consumption of antimicrobials significantly increased in the ICU-COVID group compared to the other two cohorts. In parallel with these data, there is an increase in DDD/100 bed days for meropenem, piperacillin/tazobactam, colistin, vancomycin and caspofungin in the ICU-COVID group (Table 5).

Costs for antimicrobials increased in ICU-COVID, with particular reference to carbapenems, piperacillin/tazobactam, colistin, linezolid, tigecycline and third-generation cephalosporins, while there was no increase in ceftazidime/avibactam compared to the previous year. There was an increased use of liposomal amphotericin B in patients with COVID-19 compared to that recorded in the same period in patients without COVID-19 and in those hospitalized in 2019. The ICU-COVID expenditure on antimicrobials amounted to EUR 58,264, an increase of $341 \%$ and $605.1 \%$ compared to costs in the same period of 2019 and 2020 in the ICU-2019 group and in the ICU-noCOVID group, respectively (Table 6). 
Table 5. DDD/100 bed days.

\begin{tabular}{lccc}
\hline & $\begin{array}{c}\text { ICU-2019 } \\
\text { March-April 2019 }\end{array}$ & $\begin{array}{c}\text { ICU-noCOVID } \\
\text { March-April 2020 }\end{array}$ & $\begin{array}{c}\text { ICU-COVID } \\
\text { March-April 2020 }\end{array}$ \\
\cline { 2 - 4 } & Patients $(\boldsymbol{n}=\mathbf{5 8})$ & Patients $(\boldsymbol{n}=\mathbf{4 7})$ & Patients $(\boldsymbol{n}=\mathbf{2 8})$ \\
\hline Amoxicillin/clavulanic acid & 84.5 & 105.8 & 6.5 \\
Azithromycin & 0 & 14.8 & 10.9 \\
Ceftriaxone & 9.5 & 18.1 & 2 \\
Colistin & 0 & 0 & 5.9 \\
Daptomycin & 3.4 & 20 & 0 \\
Meropenem & 7.9 & 23 & 31.9 \\
Piperacillin/tazobactam & 14.3 & 37.6 & 44.7 \\
Vancomycin & 0 & 11.5 & 17.1 \\
Caspofungin & 10 & 8.2 & 22.5 \\
Fluconazole & 1.7 & 32.9 & 0 \\
Voriconazole & - & - & - \\
\hline
\end{tabular}

DDD: defined daily dose.

Table 6. Costs of specific antibiotic drugs in three ICU cohorts.

\begin{tabular}{|c|c|c|c|}
\hline & ICU-2019 & ICU-noCOVID & ICU-COVID \\
\hline & Patients $(n=58)$ & Patients $(n=47)$ & Patients $(n=28)$ \\
\hline Aminoglycosides & EUR 51 & EUR 45 & EUR 17 \\
\hline Amoxicillin/clavulanic acid & EUR 862 & EUR 690 & EUR 87 \\
\hline Carbapenems & EUR 259 & EUR 544 & EUR 1268 \\
\hline Ceftazidime/avibactam & EUR 3256 & EUR 0 & EUR 2442 \\
\hline Ceftolozane/tazobactam & EUR 774 & EUR 0 & EUR 0 \\
\hline Cephalosporins I-II & EUR 0 & EUR 67 & EUR 0 \\
\hline Cephalosporins III & EUR 248 & EUR 283 & EUR 616 \\
\hline Fluoroquinolones & EUR 51 & EUR 46 & EUR 0 \\
\hline Glycylcyclines & EUR 752 & EUR 280 & EUR 840 \\
\hline Glycopeptides & EUR 0 & EUR 134 & EUR 335 \\
\hline Lincosamides & EUR 64 & EUR 96 & EUR 0 \\
\hline Lipopeptides & EUR 629 & EUR 709 & EUR 0 \\
\hline Macrolides & EUR 233 & EUR 361 & EUR 276 \\
\hline Nitroimidazoles & EUR 54 & EUR 54 & EUR 0 \\
\hline Linezolid & EUR 231 & EUR 144 & EUR 867 \\
\hline Penicillin & EUR 15 & EUR 0 & EUR 0 \\
\hline Piperacillin/tazobactam & EUR 1020 & EUR 1079 & EUR 3259 \\
\hline Colistin & EUR 0 & EUR 0 & EUR 210 \\
\hline Sulfonamides & EUR 0 & EUR 4 & EUR 0 \\
\hline Liposomal amphotericin B & EUR 0 & EUR 0 & EUR 24,956 \\
\hline Triazoles & EUR 1 & EUR 47 & EUR 0 \\
\hline Echinocandins & EUR 8586 & EUR 5046 & EUR 23,091 \\
\hline Tot. & EUR 17,086 & EUR 9629 & EUR 58,264 \\
\hline
\end{tabular}

The highest costs were found in the ICU-COVID ward for liposomal amphotericin B and echinocandins.

The increase in costs was strictly related to the increased consumption of antimicrobials and not to the increase in direct costs or the reduction in drug supply affected by the lockdown.

\section{Discussion}

We studied the microbial culture findings to evaluate the frequency of isolated bacteria and fungi in different specimens obtained from patients hospitalized in ICUs with or without COVID-19-positive patients between 6 March and 30 April 2020 and in the same ICU from March to April 2019. Mortality due to secondary infections in COVID-19 patients in Wuhan was reported to be $16 \%$ hospitalized patients [28].

The incidence rate of BSIs appears significantly higher compared to those concerning the other two cohorts. BSIs constituted the majority of secondary infections, with $71 / 206$ positive cultures from the central venous blood system and 48/198 positive cultures from the peripheral venous blood system $(p<0.05)$. We documented a higher rate of 
Gram-positive isolates in blood cultures. These findings may reflect a high burden of catheter-associated blood infections, in particular those due to Staphylococcus epidermidis.

No difference of positive cultures in respiratory and urinary specimens in three cohorts was shown.

Lower respiratory tract infections were documented for 88/145 samples of respiratory tract cultures, mainly due to Candida spp. and Gram-negative bacteria. The identification of Gram-negative organisms is consistent with the types of pathogens frequently associated with hospital-acquired pneumonia (HAP) in ICUs as a complication of ICU care in both pre-COVID-19 periods [29] and during the pandemic [30-33].

The large reduction of cultures from the lower respiratory tract compared to microbial isolates from the upper respiratory tract allows us to hypothesize that yeast and bacteriumpositive respiratory tract cultures were mostly likely due to contamination/colonization of the respiratory devices other than possible commensal microorganisms of the upper respiratory tract.

These data could be related to multiple device management in a pandemic setting which may have reduced the adherence to strict aseptic procedures, especially in critically ill patients managed outside the ICU or in an overcrowded ICU. Furthermore, the proper use of personal protective equipment can be challenging and can lead to lower compliance with aseptic techniques in the management of intravascular devices, which was also favored by the recruitment of new emergency health personnel. Our results are consistent with other reports from similar cohorts where a higher frequency of BSIs was reported but differ from those of a higher percentage of Gram-negative in positive respiratory cultures revealed in our study [34].

Overall, the most frequently isolated microorganisms in an ICU-COVID ward compared to the other two cohorts in our study were Acinetobacter baumannii, Enterococcus faecium, E. faecalis, S. epidermidis, Stenotrophomonas maltophilia, Candida albicans and C. tropicalis. Our data showed that increased numbers of microbial analyses and positive cultures were matched by an increased consumption of antimicrobial drugs, calculated as expenditure per drug and as DDD/100 bed days. The overall expenditure on antimicrobials between March and April 2020 in the ICU-COVID ward was EUR 58,264 compared to EUR 9629 and EUR 17,086 spent in the ICU-noCOVID and ICU-2019 wards in 2020 and 2019, respectively.

The most used drugs were piperacillin/tazobactam (44.7 DDD/bed days), meropenem (31.9 DDD/bed days), caspofungin (22.5 DDD/bed days), vancomycin (17.1 DDD/bed days) and colistin (5.9 DDD/bed days).

The finding of an expenditure for liposomal amphotericin B in the COVID-ICU ward that is not comparable with the other two cohorts confirms the data of a higher frequency of fungal isolations in patients with COVID-19, in agreement with what was reported by other authors [35-37].

These data show that in the course of the SARS-CoV-2 pandemic, there was a greater consumption of antibiotics but also an inappropriate dosage, calculated as DDD/100 days of hospitalization, in comparison with what was demonstrated in the ICU-2019 ward in 2019 and in the ICU-noCOVID ward in 2020. Other reports did not support the routine use of antibiotics in the management of COVID-19 co-infections because of little evidence of bacterial co-infections [38]. Our experience suggests that antimicrobial stewardship strategies should be targeted to limiting unnecessary antimicrobial employment during the pandemic [39].

Our study has some limitations. First of all, the limited period examined did not allow a numerically larger sample to be examined. The study was primarily aimed at defining the number of microbial tests required by an intensive care unit during the SARS-CoV-2 pandemic. These data were then compared with the microbial analyses carried out by the same SARS-CoV-2-negative ICU in the same periods of 2020 and 2019. Second, data regarding empirical antimicrobial use were not available, limiting the assessment of its impact on the development of secondary infections. Third, it was impossible to distinguish 
the persistence of positivity of the microbial analyses from the incorrect frequent repetition of the analyses for each patient, which can lead to an overestimation of infections.

\section{Conclusions}

Co-infections are possible in COVID-19-positive patients hospitalized in ICUs. Our study is based on a limited number of microbiological data collected in a short period. The increase in culture analyses performed in positive COVID-19 patients admitted in an ICU ward compared to patients without SARS-CoV-2 infection leads to the hypothesis of an excessive use of microbial analyses with numerous and frequent sampling. It remains of major importance to distinguish colonization from infection. Their misunderstanding is a major cause of inappropriate antimicrobial therapy in the absence of further clinical data that can confirm bacterial or yeast co-infection.

Our study shows how important it is to have an antimicrobial stewardship program which is useful in pandemic emergencies, such as the current one, in order to contribute to a better use of economic resources particularly in regard to those for laboratory analyses and drugs.

The need to implement responsible antimicrobial stewardship is confirmed in order to limit unnecessary use of antimicrobials and limit the trend of antibiotic resistance.

Author Contributions: Conceptualization, R.C. and C.A.V.; methodology, R.C., B.Q., A.M. and D.S.; investigation, E.M., S.S., R.R., M.L., A.B. and R.P.; data curation, D.S.; writing-original draft preparation, R.C.; writing-review and editing, R.C. All authors have read and agreed to the published version of the manuscript.

Funding: This research received no external funding.

Institutional Review Board Statement: The study was conducted according to the guidelines of the Declaration of Helsinki. Ethical review and approval were waived for this study, due to limitation of microbiological data without any reference to people.

Informed Consent Statement: Not applicable.

Data Availability Statement: The data presented in this study are contained within the article and they are available on a reasoned request by contacting the reference author.

Acknowledgments: The authors sincerely thank all the technicians of the Clinical Microbiology Laboratory and the nurses and technicians of the pharmacy of the University Hospital "S. Anna" of Ferrara.

Conflicts of Interest: The authors declare no conflict of interest.

\section{References}

1. Morris, D.E.; Cleary, D.W.; Clarke, S.C. Secondary bacterial infections associated with influenza pandemics. Front. Microbiol. 2017, 8, 1041. [CrossRef] [PubMed]

2. Assiri, A.; Al-Tawfiq, J.A.; Al-Rabeeah, A.A.; Al-Rabiah, F.A.; Al-Hajjar, S.; Al-Barrak, A.; Flemban, H.; Al-Nassir, W.N.; Balkhy, H.H.; Al-Hakeem, R.F.; et al. Epidemiological, demographic, and clinical characteristics of 47 cases of Middle East respiratory syndrome coronavirus disease from Saudi Arabia: A descriptive study. Lancet Infect. Dis. 2013, 13, 752-761. [CrossRef]

3. Yap, F.H.Y.; Gomersall, C.D.; Fung, K.S.C.; Ho, P.; Ho, O.; Lam, P.K.N.; Lam, D.T.C.; Lyon, D.J.; Joint, G.M. Increase in methicillinresistant Staphylococcus aureus acquisition rate and change in pathogen pattern associated with an outbreak of severe acute respiratory syndrome. Clin. Infect. Dis. 2004, 39, 511-516. [CrossRef]

4. Bassetti, S.; Bischoff, W.E.; Sheretz, R.J. Outbreak of methicillin-resistant Staphylococcus aureus infection associated with an outbreak of severe acute respiratory syndrome. Clin. Infect. Dis. 2005, 40, 633-634. [CrossRef] [PubMed]

5. Guan, W.J.; Ni, Z.Y.; Hu, Y.; Liang, W.H.; Ou, C.Q.; He, J.X.; Liu, L.; Shan, H.; Lei, C.L.; Hui, D.S.; et al. Clinical characteristics of coronavirus disease 2019 in China. N. Engl. J. Med. 2020, 382, 1708-1720. [CrossRef]

6. Richardson, S.; Hirsch, J.S.; Narasimhan, M.; Crawford, J.M.; McGinn, T.; Davidson, K.W.; Barnaby, D.P.; Becker, L.B.; Chelico, J.D.; Cohen, S.L.; et al. Presenting characteristics, comorbidities, and outcomes among 5700 patients hospitalized with COVID-19 in the New York City Area. JAMA 2020, 323, 2052-2059. [CrossRef] [PubMed]

7. Yang, X.; Yu, Y.; Xu, J.; Shu, H.; Liu, H.; Wu, Y.; Zhang, L.; Yu, Z.; Fang, M.; Yu, T.; et al. Clinical course and outcomes of critically ill patients with SARS-CoV-2 pneumonia in Wuhan, China: A singlecentered, retrospective, observational study. Lancet Respir. Med. 2020, 8, 475-481. [CrossRef]

8. Möhlenkamp, S.; Thiele, H. Ventilation of COVID-19 patients in intensive care units. Herz 2020, 45, 329-331. [CrossRef] [PubMed] 
9. Wu, C.; Chen, X.; Cai, Y.; Zhou, X.; Xu, S.; Huang, H.; Zhang, L.; Zhou, X.; Du, C.; Zhang, Y.; et al. Risk factors associated with acute respiratory distress syndrome and death in patients with coronavirus disease 2019 Pneumonia in Wuhan, China. JAMA Intern. Med. 2020, 180, 934-943. [CrossRef] [PubMed]

10. Feng, Y.; Ling, Y.; Bai, T.; Xie, Y.; Huang, J.; Li, J.; Xiong, W.; Yang, D.; Chen, R.; Lu, F.; et al. COVID-19 with Different Severity: A Multi-center Study of Clinical Features. Am. J. Respir. Crit. Care Med. 2020, 201, 1380-1388. [CrossRef] [PubMed]

11. Siddiqi, H.K.; Mehra, M.R. COVID-19 illness in native and immunosuppressed states: A clinical-therapeutic staging proposal. J. Heart Lung Transplant. 2020, 39, 405-407. [CrossRef] [PubMed]

12. Mehta, P.; McAuley, D.F.; Brown, M.; Sanchez, E.; Tattersall, R.S.; Manson, J.J. COVID-19: Consider cytokine storm syndromes and immunosuppression. Lancet 2020, 395, 1033-1034. [CrossRef]

13. Jamilloux, Y.; Henry, T.; Belot, A.; Viel, S.; Fauter, M.; El Jammal, T.; Walzer, T.; François, B.; Sève, P. Should we stimulate or suppress immune responses in COVID-19? Cytokine and anticytokine interventions. Autoimmun. Rev. 2020, $19,102567$. [CrossRef]

14. Póvoa, H.C.C.; Chianca, G.C.; Iorio, N.L.P.P. COVID-19: An alert to ventilatorassociated bacterial pneumonia. Infect. Dis. Ther. 2020, 9, 417-420. [CrossRef]

15. Fu, Y.; Yang, Q.; Xu, M.; Kong, H.; Chen, H.; Fu, Y.; Yao, Y.; Zhou, H.; Zhou, J. Secondary bacterial infections in critical ill patients of COVID-19. Open Forum Infect. Dis. 2020, 7, ofaa220. [CrossRef] [PubMed]

16. Hughes, S.; Troise, O.; Donaldson, H.; Mughal, N.; Moore, L.S. Bacterial and fungal coinfection among hospitalised patients with COVID-19: A retrospectivecohort study in a UK secondary care setting. Clin. Microbiol. Infect. 2020, 26, 1395-1399. [CrossRef]

17. Wang, Z.; Yang, B.; Li, Q.; Wen, L.; Zhang, R. Clinical Features of 69 Cases with Coronavirus Disease 2019 in Wuhan, China. Clin. Infect. Dis. 2020, 71, 769-777. [CrossRef]

18. Du, R.H.; Liu, L.M.; Yin, W.; Wang, W.; Guan, L.L.; Yuan, M.L.; Li, Y.L.; Hu, Y.; Li, X.Y.; Sun, B.; et al. Hospitalization and Critical Care of 109 Decedents with COVID-19 Pneumonia in Wuhan, China. Ann. Am. Thorac. Soc. 2020, 17, 839-846. [CrossRef]

19. Wan, S.; Xiang, Y.I.; Fang, W.; Zheng, Y.; Li, B.; Hu, Y.; Lang, C.; Huang, D.; Sun, Q.; Xiong, Y.; et al. Clinical Features and Treatment of COVID-19 Patients in Northeast Chongqing. J. Med. Virol. 2020, 92, 797-806. [CrossRef]

20. Xia, W.; Shao, J.; Guo, Y.; Peng, X.; Li, Z.; Hu, D. Clinical and CT features in pediatric patients with COVID-19 infection: Different points from adults. Pediatr. Pulmonol. 2020, 55, 1169-1174. [CrossRef] [PubMed]

21. Elzi, L.; Babouee, B.; Vögeli, N.; Laffer, R.; Dangel, M.; Frei, R.; Battegay, M.; Widmer, A.F. How to discriminate contamination from bloodstream infection due to coagulase-negative staphylococci: A prospective study with 654 patients. Clin. Microbiol. Infect. 2012, 18, E355-E361. [CrossRef]

22. Hooton, T.M.; Bradley, S.F.; Cardenas, D.D.; Colgan, R.; Geerlings, S.E.; Rice, J.C.; Saint, S.; Schaeffer, A.J.; Tambayh, P.A.; Tenke, P.; et al. Diagnosis, prevention, and treatment of catheter-associated urinary tract infection in adults: 2009 International Clinical Practice Guidelines from the Infectious Diseases Society of America. Clin. Infect. Dis. 2010, 50, 625-663. [CrossRef] [PubMed]

23. Johansen, T.E.; Botto, H.; Cek, M.; Grabe, M.; Tenke, P.; Wagenlehner, F.M.; Naber, K.G. Critical review of current definitions of urinary tract infections and proposal of an EAU/ESIU classification system. Int. J. Antimicrob. Agents 2011, 38, 64-70. [CrossRef]

24. Stein, R.; Dogan, H.S.; Hoebeke, P.; Kočvara, R.; Nijman, R.J.; Radmayr, C.; Tekgül, S.; European Association of Urology; European Society for Pediatric Urology. Urinary tract infections in children: EAU/ESPU guidelines. Eur. Urol. 2015, 67, 546-558.

25. European Centre for Disease Prevention and Control. Point Prevalence Survey of Healthcare-Associated Infections and Antimicrobial Use in European Acute Care Hospitals; ECDC: Stockholm, Sweden, 2013.

26. European Centre for Disease Prevention and Control. Healthcare-associated infections acquired in intensive care units. In Annual Epidemiological Report for 2017; ECDC: Stockholm, Sweden, 2019.

27. Rodríguez-Baño, J.; Paño-Pardo, J.R.; Alvarez-Rocha, L.; Asensio, Á.; Calbo, E.; Cercenado, E.; Cisneros, J.M.; Cobo, J.; Delgado, O.; Garnacho-Montero, J.; et al. Programas de optimización de uso de antimicrobianos (PROA) en hospitales españoles: Documento de consenso GEIH-SEIMC, SEFH y SEMPSPH [Programs for optimizing the use of antibiotics (PROA) in Spanish hospitals: GEIH-SEIMC, SEFH and SEMPSPH consensus document]. Farm. Hosp. 2012, 36, e1-e30.

28. Ruan, Q.; Yang, K.; Wang, W.; Jiang, L.; Song, J. Clinical predictors of mortality due to COVID-19 based on an analysis of data of 150 patients from Wuhan, China. Intensiv. Care Med. 2020, 46, 846-848. [CrossRef] [PubMed]

29. Jones, R.N. Microbial etiologies of hospital-acquired bacterial pneumonia and ventilator-associated bacterial pneumonia. Clin. Infect. Dis. 2010, 51 (Suppl. 1), S81-S87. [CrossRef]

30. Zhang, H.; Zhang, Y.; Wu, J.; Li, Y.; Zhou, X.; Li, X.; Chen, H.; Guo, M.; Chen, S.; Sun, F.; et al. Risks and features of secondary infections in severe and critical ill COVID-19 patients. Emerg. Microbes Infect. 2020, 9, 1958-1964. [CrossRef] [PubMed]

31. Ripa, M.; Galli, L.; Poli, A.; Oltolini, C.; Spagnuolo, V.; Mastrangelo, A.; Muccini, C.; Monti, G.; De Luca, G.; Landoni, G.; et al. COVID-BioB study group. Secondary infections in patients hospitalized with COVID-19: Incidence and predictive factors. Clin. Microbiol. Infect. 2021, 27, 451-457. [CrossRef] [PubMed]

32. Al-Hatmi, A.M.S.; Moshin, J.; Al-Huraizi, A.; Khamis, F. COVID-19 associated invasive candidiasis. J. Infect. 2020, 82, e45-e46. [CrossRef] [PubMed]

33. Salehi, M.; Ahmadikia, K.; Badali, H.; Khodavaisy, S. Opportunistic fungal infections in the epidemic area of COVID-19: A clinical and diagnostic perspective from Iran. Mycopathologia 2020, 185, 607-611.

34. Castaldi, S.; Luconi, E.; Marano, G.; Auxilia, F.; Maraschini, A.; Bono, P.; Ungaro, R.; Bandera, A.; Boracchi, P.; Biganzoli, E. Hospital acquired infections in COVID-19 patients in sub intensive care unit. Acta Biomed. 2020, 91, e2020017. [PubMed] 
35. Guisado-Gil, A.B.; Infante-Domínguez, C.; Peñalva, G.; Praena, J.; Roca, C.; Navarro-Amuedo, M.D.; Aguilar-Guisado, M.; Espinosa-Aguilera, N.; Poyato-Borrego, M.; Romero-Rodríguez, N.; et al. On behalf of the prioam team. Impact of the COVID-19 Pandemic on Antimicrobial Consumption and Hospital-Acquired Candidemia and Multidrug-Resistant Bloodstream Infections. Antibiotics 2020, 9, 816. [CrossRef]

36. Kokkoris, S.; Papachatzakis, I.; Gavrielatou, E.; Ntaidou, T.; Ischaki, E.; Malachias, S.; Vrettou, C.; Nichlos, C.; Kanavou, A.; Zervakis, D. ICU-acquired bloodstream infections in critically ill patients with COVID-19. J. Hosp. Infect. 2021, 107, 95-97. [CrossRef] [PubMed]

37. Nucci, M.; Barreiros, G.; Guimarães, L.F.; Deriquehem, V.A.S.; Castiñeiras, A.C.; Nouér, S.A. Increased incidence of candidemia in a tertiary care hospital with the COVID-19 pandemic. Mycoses 2021, 64, 152-156. [CrossRef] [PubMed]

38. Lansbury, L.; Lim, B.; Baskaran, V.; Lim, W.S. Co-infections in people with COVID-19: A systematic review and meta-analysis. J. Infect. 2020, 81, 266-275. [CrossRef]

39. Beović, B.; Doušak, M.; Ferreira-Coimbra, J.; Nadrah, K.; Rubulotta, F.; Belliato, M.; Berger-Estilita, J.; Ayoade, F.; Rello, J.; Erdem, H. Antibiotic use in patients with COVID-19: A 'snapshot' Infectious Diseases International Research Initiative (ID-IRI) survey. J. Antimicrob. Chemother. 2020, 75, 3386-3390. [CrossRef] [PubMed] 